www.jmscr.igmpublication.org

Impact Factor (SJIF): 6.379

Index Copernicus Value: 79.54

ISSN (e)-2347-176x ISSN (p) 2455-0450

crossrefDOI: https://dx.doi.org/10.18535/jmscr/v6i12.133

Journal Of Medical Science And Clinical Research

IGM Publication

An Official Publication of IGM Publication

\title{
A Study on Clinico-Radiological Correlation of Pneumonia in Paediatric Age Group Patients ( 2 months-5 years) in a Tertiary Medical Institute
}

\author{
Muhammad Bin Selim Halder ${ }^{1}$, Moksuda Khatun ${ }^{2}$, Arun Kumar De \\ ${ }^{1}$ Junior Resident, Paediatrics, Midnapore Medical College and Hospital \\ ${ }^{2}$ Junior Resident, Paediatrics, Medical College and Hospital, Kolkata \\ ${ }^{3}$ Professor, Paediatrics, Midnapore Medical College and Hospital \\ Department of Paediatrics, Midnapore Medical College and Hospital, West Bengal, India
}

\begin{abstract}
Aim: The objective of the study is to find out the correlation between clinical diagnosis of pneumonia and its radiological changes .

Material and Methods: One fifty and two children aged 2months to 5 years, admitted at the department of Paediatrics, Midnapore Medical College and Hospital, West Bengal, from April 2016 to March 2017 with tachypnoea and/or chest retraction were included in the study. Children with known cardiovascular disease, asthma, tuberculosis and neurological disorders were excluded. Detailed clinical history taking, meticulous clinical examinations were performed and Chest $x$-rays were taken on admission for all included patients.

Result: Sensitivity, Specificity, PPV, NPV of tachypnoea was $98.06 \%, 24.49 \%, 73.19 \%$ and $85.71 \%$ respectively. Sensitivity, specificity, PPV, NPV of grunting were calculated to be $9.70 \%, 100 \%, 100 \%$ \& $34.50 \%$ respectively. Fever, cough and tachypnoea together were present in $96(63.16 \%)$ children. Sensitivity and specificity of presence of fever, cough, and tachypnoea were $76.70 \%$ and $65.31 \%$; PPV and NPV were calculated to be $82.29 \%$ and $57.14 \%$ respectively.

Conclusion: Presence of Tachypnoea was the most sensitive parameter to diagnosis pneumonia. Grunting was the most specific parameter and had highest positive predictive value in respect of radiologically confirmed pneumonia.

Keywords: Pneumonia, Tachypnoea, Grunting.
\end{abstract}

\section{Introduction}

Pneumonia is a leading cause of death globally among children younger than five years of age and accounts for an estimated 1.2 million (18\% total) deaths annually. ${ }^{1,2}$ The number of childhoodrelated deaths from pneumonia is approximately 2000 fold higher in developing countries than in developed countries. ${ }^{3}$ The accurate diagnosis of community acquired pneumonia is an important clinical issue even though it remain difficult. ${ }^{4}$ Evidence suggests that pneumonia in under-five children may be over-diagnosed and its prevalence may be over-estimated. ${ }^{5}$ Tachypnoea, widely used as clinical predictor of pneumonia can be caused by many other conditions like any form of febrile illness, apprehension, hyper-active airway diseases, acute bronchiolitis etc. WHO guideline recommended that the respiratory rate should be measured when the child is afebrile, calm or feeding but these may not be always be possible in busy setting. ${ }^{6}$ 
The definitive final diagnosis of pneumonia require isolation of causative agent. ${ }^{7}$ Culture of sputum is of little value in the diagnosis of pneumonia in young children. Blood culture results are positive only in $10 \%$ of children with pneumococcal pneumonia. Percutaneous lung aspiration is invasive and not routinely performed. Therefore chest $\mathrm{x}$-ray can give useful information about the presence of pneumonia. $^{8}$ In daily practice, physicians require high quality (high sensitivity, specificity and predictive values and appropriate likelihood ratios) diagnostic indicators to differentiate pneumonia from other lower respiratory infections like bronchiolitis or bronchitis. This would help the clinician to avoid additional tests like radiography and blood culture, eliminate the misuse of antibiotics and limit the unnecessary hospitalization of patients. ${ }^{7}$

\section{Aims and objectives}

To find out the correlation between clinical diagnosis of pneumonia and its radiological changes.

\section{Materials and Methods}

A prospective cross-sectional hospital based single centre observational study was conducted an the department of Paediatrics, Midnapore Medical College and Hospital, West Medinipur, West Bengal, from April 2016 to March 2017. One fifty and two children aged 2months to 5 years, admitted at the department of Paediatrics with tachypnoea and/or chest retraction (clinical diagnosis of pneumonia), were included in the study. Children with known cardiovascular disease, asthma, tuberculosis and neurological disorders were excluded. Detailed clinical history taking, meticulous clinical examinations were performed and Chest x-rays were taken on admission for all included patients. The clinical variables in this study were fever, cough, tachypnoea, chest retraction, nasal flaring, grunting, diminished breath sound, crackles, wheeze and cyanosis. Radiological pneumonia was defined as parenchymal opacity with smooth outline with or without air-bronchogram in any part of lung field

\section{Results and Analysis}

Out of 152 children included in the study 79 $(51.97 \%)$ were male and $73(48.03 \%)$ were female. Radiological evidence of pneumonia was present in 103(67.76\%) children.

Table 1: Clinical characteristics of study subjects with pneumonia $(n=152)$

\begin{tabular}{|l|c|}
\hline Clinical variables & Percentage (\%) \\
\hline Fever & $86.18 \%$ \\
\hline Cough & $76.32 \%$ \\
\hline Tachypnoea & $90.79 \%$ \\
\hline Chest retraction & $59.21 \%$ \\
\hline Nasal flaring & $46.05 \%$ \\
\hline Grunting & $06.58 \%$ \\
\hline Diminished breath sound & $23.03 \%$ \\
\hline Crackles & $83.55 \%$ \\
\hline Wheeze & $51.32 \%$ \\
\hline Cyanosis & $17.76 \%$ \\
\hline Tachypnoea \& chest retraction & $50.00 \%$ \\
\hline Tachypnoea \& grunting & $06.58 \%$ \\
\hline Tachypnoea \& diminished breath sound & $23.03 \%$ \\
\hline Tachypnoea \& cyanosis & $17.76 \%$ \\
\hline Fever,cough \& tachypnoea & $63.16 \%$ \\
\hline Tachypnoea,chest retraction\& nasal flaring & $40.13 \%$ \\
\hline Tachypnoea,chest retraction\& crackles & $48.68 \%$ \\
\hline
\end{tabular}

Table 2: Summary of different clinical variables in patient having radiological evidence of pneumonia $(n=103)$

\begin{tabular}{|l|c|c|c|c|c|}
\hline Clinical variables & $\begin{array}{c}\text { Sensitivity } \\
(\%)\end{array}$ & $\begin{array}{c}\text { Specificity } \\
(\%)\end{array}$ & $\begin{array}{c}\text { PPV } \\
(\%)\end{array}$ & $\begin{array}{c}\text { NPV } \\
(\%)\end{array}$ & P-value \\
\hline Fever (n=91) & 88.34 & 18.37 & 69.47 & 42.86 & 0.3158 \\
\hline Cough(n=83) & 80.58 & 32.65 & 71.55 & 44.44 & 0.1016 \\
\hline Tachypnoea (n=101) & 98.06 & 24.49 & 73.19 & 85.71 & 0.0001 \\
\hline Chest retraction (n=68) & 66.02 & 55.10 & 75.56 & 43.55 & 0.0212 \\
\hline Nasal flaring (n=60) & 58.25 & 79.59 & 85.71 & 47.56 & 0.0001 \\
\hline Grunting (n=10) & 09.71 & 100.00 & 100.00 & 34.51 & 0.0309 \\
\hline Diminished breath sound (n=32) & 31.07 & 93.88 & 91.43 & 39.32 & 0.0004 \\
\hline Crackles (n=92) & 89.32 & 28.57 & 72.44 & 56 & 0.0091 \\
\hline Wheeze (n=60) & 58.25 & 63.27 & 76.92 & 41.89 & 0.0154 \\
\hline Cyanosis (n=23) & 22.33 & 91.84 & 85.19 & 36.00 & 0.0404 \\
\hline
\end{tabular}

PPV: Positive Predictive Value, NPV: Negative Predictive Value 
Sensitivity \& Specificity of tachypnoea were 98.06\% and $24.49 \%$ respectively; PPV \& NPV were $73.19 \%$ and $85.71 \%$ respectively. Sensitivity and specificity of grunting were calculated to be $9.70 \%$ and $100 \%$ respectively.PPV \&NPV were calculated to be $100 \%$ \& $34.50 \%$ respectively.

Table3: Different clinical variable in combination in patients having radiological evidence of pneumonia $(\mathrm{n}=103)$

\begin{tabular}{|l|c|c|c|c|c|}
\hline Clinical variables & $\begin{array}{c}\text { Sensitivity } \\
(\%)\end{array}$ & $\begin{array}{c}\text { Specificity } \\
(\%)\end{array}$ & $\begin{array}{c}\text { PPV } \\
(\%)\end{array}$ & $\begin{array}{c}\text { NPV } \\
(\%)\end{array}$ & P-value \\
\hline Tachypnoea \&chest retraction (n=66) & 64.08 & 75.51 & 84.62 & 50.00 & 0.0001 \\
\hline Tachypnoea \& grunting (n=10) & 09.71 & 100.00 & 100.00 & 34.51 & 0.0309 \\
\hline $\begin{array}{l}\text { Tachypnoea \& diminished breath sound } \\
\text { (n=32) }\end{array}$ & 31.07 & 93.88 & 91.43 & 39.32 & 0.0004 \\
\hline Tachypnoea \& cyanosis (n=23) & 22.33 & 91.84 & 85.19 & 36.00 & 0.0404 \\
\hline Fever,cough and tachypnoea (n=79) & 76.70 & 65.31 & 82.29 & 57.14 & 0.0001 \\
\hline $\begin{array}{l}\text { Tachypnoea,nasal flaring \& chest } \\
\text { retraction (n=54) }\end{array}$ & 52.43 & 85.71 & 88.52 & 46.15 & 0.0001 \\
\hline $\begin{array}{l}\text { Tachypnoea,chest retraction and crackles } \\
\text { (n=64) }\end{array}$ & 62.14 & 79.59 & 86.49 & 50.00 & 0.0001 \\
\hline
\end{tabular}

PPV: Positive Predictive value, NPV: Negative Predictive Value

Sensitivity and specificity of the presence of both tachypnoea and grunting were calculated to be 9.71\% and $100 \%$ respectively; PPV and NPV were $100 \%$ and $34.51 \%$ respectively. Fever, cough and tachypnoea together were present in 96 $(63.16 \%)$ children. Sensitivity and specificity of presence of fever, cough, and tachypnoea were calculated to be $76.70 \%$ and $65.31 \%$; PPV and NPV were calculated to be $82.29 \%$ and $57.14 \%$ respectively.

\section{Discussion}

In the present study it was found that presence of tachypnoea was statistically significant in respect of radiological pneumonia $(\mathrm{P}=.0001)$. Radiological pneumonia was present in $73.19 \%$ children who had tachypnoea. In this study tachypnoea was found to be most sensitive parameter with sensitivity $98.06 \%$ and specificity $24.49 \%$. In a study conducted by Al-Dabbagh SA et al sensitivity and specificity of tachypnoea were found to be $85.7 \%$ and $48.55 \%$ respectively ${ }^{7}$. Lozano JM et al concluded in their study that best single predictor of the presence of pneumonia is a high respiratory rate ${ }^{9}$. Mulholland EK et al also found similar sensitivity of tachypnoea $(81 \%)$ in their study ${ }^{10}$. In a study conducted by Palaflox M et al in Mexican social security institute sensitivity of tachypnoea was found to be $74 \%^{11}$.PPV of tachypnoea was found to be $73.19 \%$. Dai Y et al also found similar PPV of tachypnoea (74.5\%) in their study ${ }^{12}$.

In the present study it was found that presence of crackles was statistically significant in respect of radiologically confirmed pneumonia $(\mathrm{P}=0.0091)$. Lozano JM et al also showed in their study that presence of crackle was statistically significant in respect of radiological pneumonia ${ }^{9}$. In another study conducted by Gupta D et al in India, crepitations on auscultation of chest were found to have good correlation with radiological pneumonia $^{13}$. In the present study radiological evidence of pneumonia was present in $72.44 \%$ of children who had crackles. Sensitivity of crackles were calculated to be $89.32 \%$. This is in conformity with the finding of Bilkis MD et al Who also found similar sensitivity of crackles $(93.8 \%)^{14}$. The specificity crackles was low (28.57\%). Therefore its absence does not rule out the presence of pneumonia. This observation is comparable with study conducted by Al-Dabbagh SA et al at Mosul Medical College, Mosul, Iraq ${ }^{7}$. Grunting was present in ten $(6.58 \%)$ children. It was found to have very high specificity $100 \%$ but the sensitivity was quite low only $9.71 \%$. Lynch $\mathrm{T}$ et al also found almost similar result in their study conducted on patients aged one to 16 years ${ }^{15}$. In another study conducted by Kuti BP et al at Obafemi Awolowo University, Nigeria the sensitivity and specificity were found to be $37.5 \%$ 
and $77.94 \%$ respectively ${ }^{16}$. In their study children of age group 2 months to 5years were included. In the present study presence of diminished breath sound was found to be statistically significant in respect of radiological pneumonia $(\mathrm{P}=0.0004)$. Neuman MI et al conducted a prospective cohort study in Boston, they also found that children aged less than 5 years with presence of focal decreased breath sound were significantly more likely to have radiological pneumonia relative to children without this feature ${ }^{17}$. Sensitivity and specifity of diminished breath sound were calculated to be $31.07 \%$ \&93.88\%;PPV \& NPV were $91.43 \%$ and $39.32 \%$.In the study conducted by Al-Dabbag SA et al the specificity of diminished breath sound were $93.6 \%$, PPV and NPV were $75 \%$ and $32.6 \%$, wich corroborate with the finding of present study ${ }^{7}$.

In the present study cyanosis had sensitivity of $22.33 \%$ and specificity of $91.84 \%$.PPV and NPV of cyanosis were found to be $85.19 \%$ and 365 respectively. In the study conducted by Samim A Al-Dabbagh et al the sensitivity of cyanosis was $14.3 \%$ and the specificity was $90.9 \%$; PPV and NPV were $76.9 \%$ and $32.3 \%$ respectively ${ }^{7}$. These findings corroborate with the findings of the present study. In a study conducted by Kuti BP et al sensitivity and specificity of cyanosis were found to be $6.94 \%$ and $99.02 \%$ respectively ${ }^{16}$. Gupta D et al also found in their study that cyanosis was highly specific sign in detecting pneumonia but had low sensitivity ${ }^{13}$.In the present study wheeze was found to be statistically significant in respect of radiological pneumonia $(\mathrm{P}=0.0154)$. Sensitivity and specificity wheeze were found to be $58.25 \%$ \&63.27\%.In a study conducted by Samim A Al-Dabbagh et al sensitivity and specificity of wheeze were $61.4 \%$ and $33.3 \%$ respectively ${ }^{7}$.

In the present study chest retraction was found to be statistically significant $(\mathrm{P}=.0212)$ in respect of radiological pneumonia. Sensitivity and specificity were calculated to be $66.02 \%$ and $55.10 \%$. Mulholland EK et al conducted one prospective study in Manila, Philippines and Mbabane, Swaziland using an identical protocol in both countries. In the Philippines chest in drawing was found to have sensitivity of 0.81 and specificity of 0.77 for predicting pneumonia. In Swaziland the sensitivity was 0.77 and specificity was $0.80 .{ }^{10}$ These findings corroborate with the present study. Singhi $\mathrm{S}$ et al conducted one prospective study, in India they concluded that chest retraction and/or rapid or difficult breathing were sensitive and specific indicator of pneumonia. ${ }^{18}$ Presence of tachypnoea and chest retraction when consider together specificity increased considerably $(75.51 \%)$ but sensitivity did not improved (64.08).In the present study nasal flaring was found to be statistically significant $(\mathrm{P}=.0001)$ in respect of radiological pneumonia. Sensitivity of nasal flaring was $58.25 \%$ and specificity $79.59 \%$.PPV and NPV were $85.71 \%$ and $47.56 \%$ respectively. MahabeeGitten EM et al conducted one prospective cohort study in Cincinnati, USA. They found that in children under age 12 months nasal flaring can be used in determining which children with lower respiratory tract infection symptoms have radiographic pneumonia ${ }^{19}$.

In the present study presence of fever was found not to be statistically significant in respect of radiologically confirmed pneumonia. WHO algorithm for diagnosis of pneumonia in children also does not include fever. In previous studies there were conflicting result regarding sensitivity of fever on predicting radiological pneumonia. ${ }^{20 \text {, }}$ ${ }^{21,} 22$ In the present study presence of cough was found not to be statistically significant. WHO algorithm for diagnosis of pneumonia also does not include cough. It was found to have good sensitivity $80.58 \%$ but low specificity $32.65 \%$. S Shuba et al also found in their study that cough had good sensitivity but poor specificity ${ }^{21}$.Present study showed presence of fever, cough and tachypnoea together were found to be statistically significant $(\mathrm{P}=.0001)$. One previous study has demonstrated that use of multiple indicators at the same time in parallel (considering the presence or absence of any criterion as a positive and negative diagnosis) increases the sensitivity and reduces the false negative 
diagnosis ${ }^{23}$. Another study has shown that the use of multiple indicators serially (considering the presence or absence of any indicator as a positive or negative diagnosis respectively) maximizes the sensitivity and specificity. ${ }^{16}$

\section{Conclusion}

Tachypnoea was the most sensitive parameter to diagnosis pneumonia. Presence of crackles also had good correlation with radilogically confirmed pneumonia. Grunting was the most specific parameter and had highest positive predictive value in respect of radiologically confirmed pneumonia. Presence of diminished breath sound and cyanosis individually or in combination with tachypnoea had very high specificity and positive predictive value in respect of radiologially confirmed pneumonia.

Though presence of fever and cough individually were not statistically significant, presence of fever, cough and tachypnoea together was found to be statistically significant.

\section{References}

1. Black RE, Allen LH, Bhutta ZA, et al: Maternal and child undernutrition: global and regional exposures and health consequences. Lancet 371:243-260.2008.

2. Liu L, Johnson HL, Cousens $\mathrm{S}$, et al. Global, regional, and national causes of child mortality: an updated systemic analysis for 2010 with time trends since 2000.Lancet 379:2151-2161, 2012.

3. Wardlaw T, Salama P, White Johansson E: Pneumonia the leading killer of children, Lancet 368:1048-1050, 2006.

4. Key NK.Araújo-Neto CA, Cardoso MRA, Carvalho CMN. Characteristics of radiologically diagnosed pneumonia in under-5 children in Salvador, Brazil.Indian paediatrics $2011 ;(48): 873-7$.

5. Rebecca N, James KT, Grace N, Marianne SO. Asthma and pneumonia among children less than five years with acute respiratory symptoms in Mulago Hospital,
Uganda: Evidence of under diagnosis of Asthma. PLos ONE 2013;8(11):1-9.

6. Hazir T, Nisar YB,Qazi SA, Khan SF, Raza M, Zameer S, et al. Chest radiography in children aged 2-59 months diagnosed with non severe pneumonia as defined by World Health Organization: Descriptive multicentre study in Pakistan.BMJ 2006;333(7569):1-4.

7. Al-Dabbagh SA, Al-Zubaidi SN.The validity of clinical criteria in predicting pneumonia among children under 5 years of age.J Fam Community Med 2004;(11):11-6

8. Eldin K, Salih MA,Wahb OA, Ibrahim SA. Radiological finding in severe pneumonia in children 1-59 months in a children's Hospital, Khartoum, Sudan. Pediatr Therapeut 2012 Mar;2(3):1-3

9. Lozano JM, Steinhoff M, Ruiz JG, Mesa ML, Martinez N, Dussan B.Clinical predictors of acute radiological pneumonia and hypoxaemia at high altitude.Arch Dis Child 1994 Oct;71(4):323-7.

10. Mulholland EK,Simoes EA,Costales MO, McGrath EJ, Manalac EM,Gove S. Standardized diagnosis of pneumonia in developing countries. Pediatr Infect Dis J 1992 Feb;11(2):77-81.

11. Palafox M, Guiscafré H,Reyes H, Muñoz O.Diagnostic value of tachypnoea in pneumonia defined radiologically. Arch Dis Child 2000;(82):41-5.

12. Dai Y,Foy HM,Zhu Z,Chen B,Tong F. Respiratory rate and signs in roentgen graphically confirmed pneumonia among children in China. Pediatr Infect Dis J 1995 Jan;14(1)48-50.

13. Gupta D.Mishra S, Chaturvedi P.Fast breathing in the diagnosis of pneumonia-a reassessment Trop Pediatr 1996 Aug; 42(4):196-9.

14. Bilkis MD, Gorgal N, Carbone M, Vazquez M, Albanese P, Branda MC, et al. Validation and development of a clinical prediction rule in clinically suspected 
community-acquired pneumonia. Pediatr Emerg Care 2010 Jun;26(6):399-405.

15. Lynch T,Platt R, Gouin S, Larson C, Patenaude Y.Can We Predict Which Children With Clinically Suspected Pneumonia Will Have The Presence of Focal Infiltrates on Chest radiographs? Pediatrics 2004;113(3 Pt 1):e1869.Available from PubMed.

16. Kuti BP, Adegoke SA, Oyelami OA.Can we predict which children with clinical pneumonia will have radiologic findings on chest radiograph? World Journal of Medicine and Medical science 2014 Mar;2(3):1-12.

17. Neuman MI,Scully KJ,Kim D,Shah S,Bachur RG.Physician assessment of the likelihood of pneumonia in a pediatric emergency department.Pediatric Emerg Care 2010 Nov;26(11):817-22.

18. Singhi S, Dhawan A,Kataria S,Walia BN. Validity of clinical signs for the identification of pneumonia in children. Ann Trop Pediatr 1994;14(1):53-8.

19. Gittens MEM, Phelan GJ, Brody AS, Donnelly LF, Bracey SE, Duma EM, et al.Identifying children with pneumonia in the emergency department.Clin Pediatr 2005 Jun;44(5):427-35

20. Zukin DD, Hoffman JR, Cleveland RH, Kushner DC, Herman TE.Correlation of pulmonary signs and symptoms with chest radiographs in the pediatric age group. Ann Emerg Medicine 1986 Jul; 15(7):7926.

21. Shuba S, Amullya S, Raghu MB,Kumar R. Correlation of clinincal pneumonia with radiological changes in children less than 5 years. Available from: URL: www.iapindia.org/files/abstracts 2006/RESP.doc.

22. Cornelius W, Van N, Lateef TM, Mathwes B. Reducing exposer in the ED. Pediatrics 2009 Jul;(1):1-3.
23. Harari M, Shann F, Spooner V, Meisner S, Carney M, Campo DJ. Clinical signs of pneumonia in children. Lancet 1991 oct 12;338(8772):928-30. 